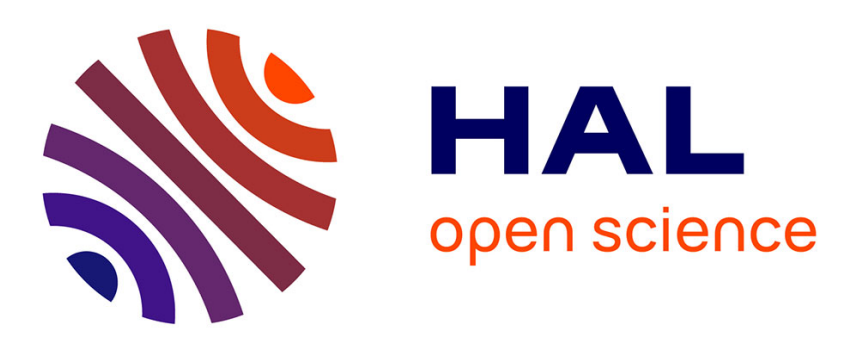

\title{
Unstructured PEEC Method with the use of Surface Impedance Boundary Condition
}

Gérard Meunier, Q.-A Phan, Olivier Chadebec, J M Guichon, B Bannwarth, $\mathrm{R}$ Torchio

\section{- To cite this version:}

Gérard Meunier, Q.-A Phan, Olivier Chadebec, J M Guichon, B Bannwarth, et al.. Unstructured PEEC Method with the use of Surface Impedance Boundary Condition. COMPEL: The International Journal for Computation and Mathematics in Electrical and Electronic Engineering, 2020, 39 (5), pp.1017-1030. 10.1108/COMPEL-01-2020-0023 . hal-02991208

\section{HAL Id: hal-02991208 \\ https://hal.science/hal-02991208}

Submitted on 5 Nov 2020

HAL is a multi-disciplinary open access archive for the deposit and dissemination of scientific research documents, whether they are published or not. The documents may come from teaching and research institutions in France or abroad, or from public or private research centers.
L'archive ouverte pluridisciplinaire HAL, est destinée au dépôt et à la diffusion de documents scientifiques de niveau recherche, publiés ou non, émanant des établissements d'enseignement et de recherche français ou étrangers, des laboratoires publics ou privés. 


\title{
Unstructured PEEC Method with the use of Surface Impedance Boundary Condition
}

\author{
G. Meunier ${ }^{1}$, Q.-A. Phan ${ }^{1}$, O. Chadebec ${ }^{1}$, J.M. Guichon ${ }^{1}$, B. Bannwarth ${ }^{1}$, R. Torchio ${ }^{1,2}$ \\ ${ }^{1}$ Univ. Grenoble Alpes, CNRS, Grenoble INP, G2Elab, F-38000 Grenoble, France \\ ${ }^{2}$ Dipartimento di Ingegneria Industriale, Università degli Studi di Padova, 35131 Padova, Italy \\ gerard.meunier@g2elab.grenoble-inp.fr
}

\begin{abstract}
An unstructured-PEEC method for modelling electromagnetic regions with surface impedance condition (SIBC) is proposed. Two coupled circuits representations are used for solving both electric and/or magnetic effects in thin regions discretized by a finite element surface mesh. The formulation is applied in the context of low frequency problems with volumic magnetic media and coils. Non simply connected regions are treated with fundamental branch independent loop matrices coming from the circuit representation.
\end{abstract}

Purpose - An unstructured PEEC (Partial Element Equivalent Circuit) method for modelling electromagnetic regions with Surface Impedance Boundary Condition (SIBC) is proposed

Design/Methodology/Approach - Thanks to the use of Withney face elements, two coupled circuits representations are used for solving both electric and/or magnetic effects in thin regions discretized by a finite element surface mesh. The air is not meshed.

Finding - The new surface impedance formulation enables the modeling of volume conductive regions in order to efficiently simulate various devices with only a surface mesh.

Research limitations implications - The propagation effects are not taken into account in the proposed formulation

Originality/Value - The formulation is original and is efficient for modelling non simply connected conductive regions with the use of SIBC. The unstructured PEEC SIBC formulation has been validated in presence of volume magnetic nonconductive region and compared with a SIBC FEM approach. The computational effort is considerably reduced in comparison with volume approaches.

Keywords - Volume Integral Formulation, Unstructured PEEC, Surface Impedance Condition, Losses.

1

\section{Introduction}

A major interest in using Volume Integral Methods for 3D magnetic and electric fields analysis is that the air region does not need to be meshed. Moreover, the development of new efficient matrix compression algorithms (e.g. FMM, ACA, which greatly improve memory storage and resolution time of fully dense matrix systems) renewed the interest of solving Maxwell's equations with integral methods based on the Green's function. In this work, we propose a new formulation dedicated to electromagnetic regions treated by the surface impedance condition [1]. This work is an extension of a previous volume integral approach based on 3D facet interpolations of the current density and of the magnetic flux density [2], [8] and SIBC approaches in the case of conductive but nonmagnetic regions [11]. The main interest is that volume conductive regions only require a surface mesh and that the air is not discretized (unlike finite element approaches combined with SIBC). In others words, only the interface between active and air region need to be meshed.

This approach can be compared to Boundary Equation Method (BEM) which also requires the surface mesh of the interface between air and active regions. Some formulations have already been developed in order to take account conductive and magnetic regions [9][10]. These approaches are powerful and are more general than the SIBC formulation presented in this work because they

\footnotetext{
${ }^{1}$ The paper has been presented at the ISEF conference
} 
are not based on any approximation. However, BEM implementations are not straightforward and their performances are penalized by the need to integrate complex Green's kernels which is always a difficulty and a can be source of numerical inaccuracies. So, when the skin depth allows the use of SIBC, our integral formulation becomes very attractive in terms of computing performances. Moreover, thanks to the circuit representation, these formulations can be easily and naturally coupled with external circuits, PEEC1D cables or coil which is not straightforward in the BEM context.

In this paper, we first present fundamental equations for building the circuit approach formulation for volume con-ducting regions treated with SIBC. The proposed formulation is applied to low frequency problems (capacitive effects and propagation effects will be neglected) in presence of coils and magnetic non conducting regions.

\section{Formulation}

\subsection{Volume Integral Formulation}

Let us consider a linear magnetoharmonic problem with electromagnetic regions $\Omega$ (with current density $\mathbf{J}$ and magnetization $\mathbf{M}$ ), and source coils $\Omega_{0}$ (with imposed current density $\mathbf{J}_{0}$ ). Based on the solution of Maxwell equations, electric field $\mathbf{E}$ and magnetic field $\mathbf{H}$ can be written in terms of the Green's functions, the current density $\mathbf{J}$, and magnetization $\mathbf{M}$ by means integral expression over $\Omega$. In the frequency domain, by neglecting propagation effects, and thanks to Lorentz gauge, we have:

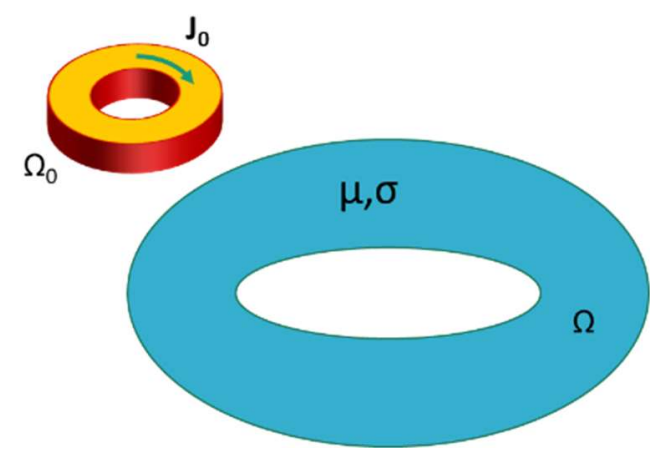

Fig. 1. Typical electromagnetic problem

$$
\begin{aligned}
\mathbf{E}(P) & =-j \omega \mathbf{A}(P)-\operatorname{grad} V \\
\mathbf{H}(P) & =\mathbf{T}(\mathrm{P})-\operatorname{grad} \varphi
\end{aligned}
$$

with 


$$
\begin{gathered}
\mathbf{T}(\mathrm{P})=\frac{1}{4 \pi}\left(\iint_{\Omega_{J}} \mathbf{J} \wedge \boldsymbol{g r a d} \frac{1}{r} d \Omega+\iint_{\Omega_{0}} \mathbf{J}_{\mathbf{0}} \wedge \boldsymbol{g r a d} \frac{1}{r} d \Omega\right) \\
\mathbf{A}(\mathrm{P})=\frac{\mu_{0}}{4 \pi}\left(\iint_{\Omega_{J}} \frac{\mathbf{J}}{r} d \Omega+\iint_{\Omega_{0}} \frac{\mathbf{J}_{\mathbf{0}}}{r} d \Omega+\iint_{\Omega_{M}} \mathbf{M} \wedge \boldsymbol{g r a d} \frac{1}{r} d \Omega\right) \\
V(P)=\frac{1}{j \omega} \frac{1}{4 \pi \varepsilon_{0}} \iint_{\Omega_{J}} \mathbf{J} \boldsymbol{g r a d} \frac{1}{r} d \Omega \\
\varphi(P)=\frac{1}{4 \pi} \iint_{\Omega_{M}} \mathbf{M} \cdot \boldsymbol{g r a d} \frac{1}{r} d \Omega
\end{gathered}
$$

In the above expressions, $\mathrm{r}$ is the distance between observation point $\mathrm{P}$ and the integration point. Volume Integral Equations [2] are then obtained by matching (1) and (2) with constitutive relationships $\mathrm{E}(\mathrm{J})$ and $\mathrm{H}(\mathrm{M})$ inside $\Omega_{J}$ and $\Omega_{M}$, which, in the case of linear properties, are :

$$
\begin{gathered}
\mathbf{E}=\frac{1}{\sigma} \mathbf{J} \\
\mathbf{H}=\frac{1}{\mu_{r}-1} \mathbf{M}
\end{gathered}
$$

where $\sigma$ is the conductivity and $\mu=\mu 0 \mu \mathrm{r}$ is the permeability. Different formulations can be obtained by discretizing the regions and by choosing adequate unknowns and interpolation shape functions. The use of 2-form Whitney face interpolation for both current density $\mathrm{J}$ and magnetic flux density B leads to the unstructured PEEC method proposed in [2].

\subsection{Surface Impedance Boundary Condition (SIBC)}

We consider that the frequency is sufficiently high to use the surface impedance first order approximation [1]. Thus, we consider that the current and flux density in the volume regions are mainly tangential to the boundary and located in a thin pellicular region placed of $\Omega$. Tangential current density $\mathrm{J}$ and flux density B along the perpendicular direction $(\mathrm{z})$ of the boundary are express by :

$$
\begin{aligned}
\mathbf{J} & =\mathbf{J}_{\boldsymbol{s}} e^{-(1+j) \frac{Z}{\delta}} \\
\mathbf{B} & =\mathbf{B}_{\mathbf{s}} e^{-(1+j) \frac{Z}{\delta}}
\end{aligned}
$$

$\mathbf{J}_{\mathbf{s}}$ and $\mathbf{B}_{\mathbf{s}}$ being the tangential surface current and flux densitiy on $\Gamma$ (the external boundary of $\Omega$ ) respectively, whereas $\delta$ is the skin depth. Tangential surface current $\mathbf{K}$ (in A/m) and surface flux density $\boldsymbol{\Phi}$ (in $\mathrm{Wb} / \mathrm{m}$ ) are obtained by integrating $\mathbf{J}$ and $\mathbf{B}$ along (z) i.e.:

Then, we have:

$$
\begin{aligned}
& \mathbf{K}=\mathbf{J}_{\boldsymbol{s}} \frac{\delta}{1+j} \\
& \mathbf{\Phi}=\mathbf{B}_{\mathbf{s}} \frac{\delta}{1+j}
\end{aligned}
$$


From (1) and (2), the evaluation of electric and magnetic fields can be obtained from $\mathbf{K}$ and $\mathbf{\Phi}$ on $\Gamma$. Integral equations are then obtained by matching the values of electric and magnetic fields on the boundaries of the pellicular region. This leads to two surface integral equations with $\mathbf{K}$ and $\mathbf{\Phi}$ as variables:

$$
\begin{gathered}
\frac{1+j}{2 \sigma \delta} \mathbf{K} \approx-\operatorname{grad} V-\frac{j \omega \mu_{0}}{4 \pi} \int_{\Omega_{0}} \frac{\mathbf{J}_{\mathbf{0}}}{r} d \Omega \\
-\frac{j \omega}{4 \pi}\left(\mu_{0} \int_{\Gamma} \frac{\mathbf{K}}{r} d \Gamma+\int_{\Gamma} \frac{\mu_{r}-1}{\mu_{r}} \mathbf{\Phi} \wedge \operatorname{grad} \frac{1}{r} d \Gamma\right) \\
\frac{1+j}{2 \sigma \delta} \boldsymbol{\Phi} \approx-\operatorname{grad} \varphi+\frac{1}{4 \pi} \iint_{\Omega_{0}} \mathbf{J}_{0} \wedge \operatorname{grad} \frac{1}{r} d \Omega \\
+\frac{1}{4 \pi} \int_{\Gamma} \mathbf{K} \wedge \boldsymbol{g r a d} \frac{1}{r} d \Gamma
\end{gathered}
$$

\subsection{Use of $2 D$ face element on $3 D$ surface}

Region $\Gamma$ is discretized by surface finite element meshes (composed of triangles or quadrilaterals for instance) on which $\mathbf{K}$ and $\Phi$ are interpolated with 2D first order surface face elements:
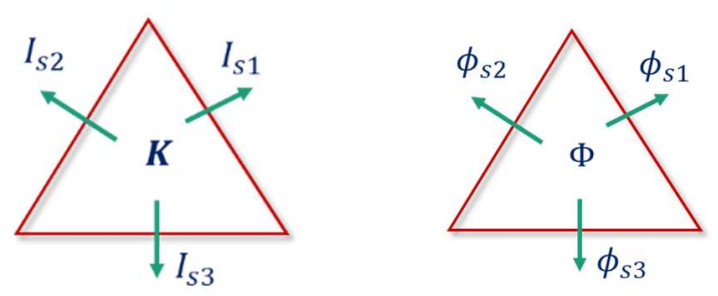

$$
\mathbf{K}=\sum_{j} \boldsymbol{w}_{s j} I_{s j}
$$$$
\boldsymbol{\Phi}=\sum_{j} \boldsymbol{w}_{s j} \phi_{s j}
$$

$\boldsymbol{w}_{\boldsymbol{s}}$ is the face function related to $\mathrm{j}$-th face, whereas $I_{s j}$ and $\phi_{s j}$ are the current (in A) and magnetic flux (in Wb) flowing through the $\mathrm{j}$-th face, respectively. It is worth notice that, with a surface mesh, face elements are associated to the edges of the mesh).

We have:

$$
\begin{gathered}
\operatorname{div} \boldsymbol{w}_{\boldsymbol{s i}}= \pm \frac{1}{s_{e}} \\
\boldsymbol{w}_{\boldsymbol{s i}} \cdot \boldsymbol{n}= \pm \frac{1}{l_{i}} \text { on face i (0 on other faces) }
\end{gathered}
$$


where $s_{e}$ is the area of the e-th element, and $l_{i}$ is the length of $\mathrm{i}$-th face (geometric edge). The signs in (5) depend on the choice of the global orientation of the face. These relations ensure the continuity of K.n and $\boldsymbol{\Phi} . \mathbf{n}$ between adjacent elements. Using (5), by considering any scalar potential $\mathrm{u}$ and thanks to divergence theorem, we obtain:

$$
\int_{\Gamma} w_{i s} \operatorname{grad} u=\Delta u_{i}
$$

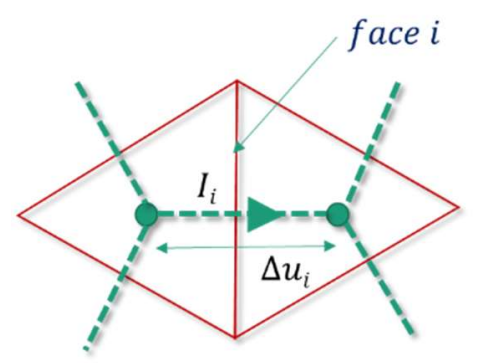

Figure 2. Primal and dual mesh (dotted lines)

where $\Delta u_{i}$ is the difference between both mean values of the potential $\mathrm{u}$ on elements sharing face $i$, as shown in Fig. 2. These properties will be useful to construct an equivalent circuit representation based on the dual mesh, as developed in the next section.

It is worth notice that the surface divergences of $\mathbf{K}$ and $\mathbf{\Phi}$ are generally different from zero. Indeed, these divergences are related to the current / flux flowing out from $\Gamma$. Thus, assuming that the faces of the elements are oriented towards the outside, we have:

$$
\begin{gathered}
\operatorname{div}_{s} \mathbf{K}=\frac{1}{s_{e}} \sum_{j} I_{s j} \\
\operatorname{div}_{s} \mathbf{\Phi}=\frac{1}{s_{e}} \sum_{j} \phi_{s j}
\end{gathered}
$$

\subsection{Unstructured PEEC model for thin regions}

Applying a Galerkin projection with facet functions $\mathrm{w}_{\mathrm{i}}$ to equations (4), and by considering (5), we get a circuit representation on the dual mesh [2]:

$$
\left\{\begin{array}{c}
\Delta V_{s} \\
\Delta \varphi_{\mathrm{s}}
\end{array}\right\}=\left\{\begin{array}{cc}
R+j \omega L & j \omega C \\
C^{\prime} & Y
\end{array}\right\}\left\{\begin{array}{c}
I_{S} \\
\phi_{S}
\end{array}\right\}+\left\{\begin{array}{c}
S \\
T
\end{array}\right\}
$$

with

$$
\begin{aligned}
R_{i j} & =\int_{\Gamma} \frac{1+j}{2 \delta} \frac{\boldsymbol{w}_{\boldsymbol{s i}} \boldsymbol{w}_{\boldsymbol{s}}}{\sigma} d \Omega \\
L_{i j} & =\frac{\mu_{0}}{4 \pi} \int_{\Gamma} \boldsymbol{w}_{\boldsymbol{s i}} \int_{\Gamma} \frac{\boldsymbol{w}_{\boldsymbol{s}}}{r} d \Gamma d \Gamma
\end{aligned}
$$




$$
\begin{gathered}
Y_{i j}=\int_{\Gamma} \frac{1+j}{2 \delta} \frac{\boldsymbol{w}_{\boldsymbol{s i}} \boldsymbol{w}_{\boldsymbol{s j}}}{\mu} d \Omega \\
C_{i j}=\frac{1}{4 \pi} \int_{\Gamma} \boldsymbol{w}_{\boldsymbol{s i}} \int_{\Gamma} \frac{\mu_{r}-1}{\mu_{r}} \boldsymbol{w}_{\boldsymbol{s j}} \wedge \boldsymbol{g r a d} \frac{1}{r} d \Gamma d \Gamma \\
C^{\prime}{ }_{i j}=\frac{1}{4 \pi} \int_{\Gamma} \boldsymbol{w}_{\boldsymbol{s i}} \int_{\Gamma} \boldsymbol{w}_{\boldsymbol{s j}} \wedge \boldsymbol{g r a d} \frac{1}{r} d \Gamma d \Gamma \\
S=\frac{j \omega \mu_{0}}{4 \pi} \int_{\Gamma} \boldsymbol{w}_{\boldsymbol{s i}} \int_{\Omega_{0}} \frac{\boldsymbol{J}_{\mathbf{0}}}{r} d \Omega d \Gamma \\
T=\frac{1}{4 \pi} \int_{\Gamma} \boldsymbol{w}_{\boldsymbol{s i}} \iint_{\Omega_{0}} \boldsymbol{J}_{\mathbf{0}} \wedge \boldsymbol{g r a d} \frac{1}{r} d \Omega d \Gamma
\end{gathered}
$$

The equivalent circuit (dual mesh) is made by the branches connecting two adjacent elements (i.e. face of primal mesh), where elements are the nodes of the dual mesh. $\left\{\Delta \mathrm{V}_{\mathrm{s}}\right\}$ and $\left\{\Delta \varphi_{\mathrm{s}}\right\}$ represent the differences between electric and magnetic potentials on the branches of the dual mesh. R and Y are sparse finite element matrices while L, C and C' are fully populated integral matrices.

In order to take into account magnetic fluxes flowing out from magnetic regions and to consider the presence of capacitive effects in the electric regions (if any), equations (5) must be completed. The value of electric and magnetic potentials on each face element of $\Gamma$ can be obtained from equations (2). Considering that $\operatorname{div} \mathbf{J}=0$ in $\Omega_{\mathrm{J}}$, we have :

$$
\iint_{\Omega_{J}} \mathbf{J} \cdot \boldsymbol{g r a d} \frac{1}{r} d \Omega=\int_{\Gamma} \mathbf{J} \cdot \boldsymbol{n}_{\Gamma} d \Gamma=\sum_{l} \int_{\Gamma_{l}} \frac{1}{s_{l}} I_{e l} d \Gamma
$$

where $I_{e l}$ is the capacitive current flowing out from element l. Moreover, we have:

$$
\iint_{\Omega_{J}} \text { M. } \boldsymbol{g r a d} \frac{1}{r} d \Omega=\frac{1}{\mu_{0}} \sum_{l} \int_{\Gamma_{l}} \frac{\mu_{r}-1}{\mu_{r}} \frac{1}{s_{l}} \phi_{e l} d \Gamma
$$

where $\phi_{e l}$ is the magnetic flux flowing out from element 1.

The average value of potentials on each element of $\Gamma$ can then be written as:

$$
\begin{gathered}
\left\{V_{e}\right\}=P\left\{I_{e}\right\} \\
\left\{\varphi_{e}\right\}=Q\left\{\phi_{e}\right\}
\end{gathered}
$$

with 


$$
\begin{gathered}
P_{k l}=\frac{1}{4 \pi \varepsilon_{0}} \int_{\Gamma_{k}} \frac{1}{s_{k}} \int_{\Gamma_{l}} \frac{1}{s_{l}} \frac{1}{r} d \Gamma d \Gamma \\
Q_{k l}=\frac{1}{4 \pi \mu_{0}} \int_{\Gamma_{k}} \frac{1}{s_{k}} \int_{\Gamma_{l}} \frac{\mu_{r}-1}{\mu_{r}} \frac{1}{s_{l}} \frac{1}{r} d \Gamma d \Gamma
\end{gathered}
$$

$\left\{\phi_{e}\right\}$ and $\left\{\mathrm{I}_{\mathrm{e}}\right\}$ are the magnetic fluxes and electric currents flowing out from the surface elements of $\Gamma$ to the air. By considering that electric and magnetic potentials are null at infinite, the electric and magnetic circuits are completed by adding branches which connect nodes (i.e. surface elements) of $\Gamma$ to the infinite (Fig. 3).

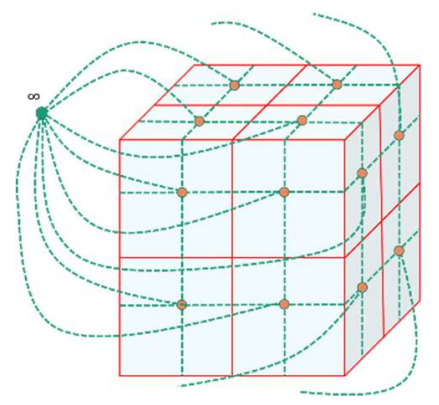

Figure. 3: Example of magnetic equivalent circuit (dotted lines) for a cubic volume region treated by SIBC. External branches allow to take into account magnetic fluxes going outside of the region. Analog circuit is used for electric circuit with or without external branches depending of the presence of capacitive effects.

Then, the complete set of equations representing the equivalent circuit interpretation of the electromagnetic problem is given by:

$$
\left\{\begin{array}{c}
\Delta V_{s} \\
\Delta V_{e} \\
\Delta \varphi_{s} \\
\Delta \varphi_{e}
\end{array}\right\}=\left\{\begin{array}{cccc}
R+j \omega L & 0 & j \omega C & 0 \\
0 & P & 0 & 0 \\
C^{\prime} & 0 & Y & 0 \\
0 & 0 & 0 & Q
\end{array}\right\}\left\{\begin{array}{c}
I_{s} \\
I_{e} \\
\phi_{s} \\
\phi_{e}
\end{array}\right\}+\left\{\begin{array}{c}
S \\
0 \\
T \\
0
\end{array}\right\}
$$

The solution can then be obtained using a circuit solver based on an independent loops search technique for instance. Fundamental circuit equations to be solved are:

$$
\begin{array}{r}
{\left[M_{I}\right]\{\Delta V\}=0 \text { with }\{\Delta V\}=\left\{\begin{array}{l}
\Delta V_{s} \\
\Delta V_{e}
\end{array}\right\}} \\
{\left[M_{\phi}\right]\{\Delta \varphi\}=0 \text { with }\{\Delta \varphi\}=\left\{\begin{array}{l}
\Delta \varphi_{s} \\
\Delta \varphi_{e}
\end{array}\right\}}
\end{array}
$$

where $\left[M_{I}\right]$ and $\left[M_{\phi}\right]$ are the branch-fundamental independent loop matrices of the electric and magnetic equivalent circuit representations, respectively. The unknowns of the system to solve are the mesh currents $\mathrm{I}_{\mathrm{M}}$ and mesh flux density $\phi_{M}$ :

$$
\{I\}=\left[M_{I}^{t}\right]\left\{I_{M}\right\} \text { with }\{I\}=\left\{\begin{array}{l}
I_{s} \\
I_{e}
\end{array}\right\}
$$




$$
\{\phi\}=\left[M_{\phi}^{t}\right]\left\{\phi_{M}\right\} \text { with }\{\phi\}=\left\{\begin{array}{c}
\phi_{s} \\
\phi_{e}
\end{array}\right\}
$$

The final system be solving is:

$$
\left[\begin{array}{cc}
{\left[M_{I}\right]\left[Z_{I}\right]\left[M_{I}^{t}\right]} & \left.\left[M_{I}\right]\left[Z_{I \phi}\right]\left[M_{\phi}^{t}\right]\right] \\
{\left[M_{\phi}\right]\left[Z_{\phi I}\right]\left[M_{I}^{t}\right]} & {\left[M_{\phi}\right]\left[Z_{\phi}\right]\left[M_{\phi}^{t}\right]}
\end{array}\right]\left\{\begin{array}{c}
I_{M} \\
\phi_{M}
\end{array}\right\}=\left\{\begin{array}{c}
U_{I} \\
U_{\phi}
\end{array}\right\}
$$

With:

$$
\begin{gathered}
{\left[Z_{I}\right]=\left[\begin{array}{cc}
R+j \omega L & 0 \\
0 & P
\end{array}\right] \quad\left[Z_{I \phi}\right]=\left[\begin{array}{cc}
j \omega C & 0 \\
0 & 0
\end{array}\right]} \\
{\left[Z_{\phi I}\right]=\left[\begin{array}{ll}
C^{\prime} & 0 \\
0 & 0
\end{array}\right] \quad\left[Z_{\phi}\right]=\left[\begin{array}{ll}
Y & 0 \\
0 & Q
\end{array}\right]} \\
\left\{U_{I}\right\}=\left[M_{I}\right]\left\{\begin{array}{l}
S \\
0
\end{array}\right\} \\
\left\{U_{\phi}\right\}=\left[M_{\phi}\right]\left\{\begin{array}{l}
T \\
0
\end{array}\right\}
\end{gathered}
$$

In the case of only conducting regions, the magnetic unknowns are not needed and the system to solve becomes:

$$
\left[M_{I}\right]\left[Z_{I}\right]\left[M_{I}^{t}\right]\left\{I_{M}\right\}=\left\{U_{I}\right\}
$$

\subsection{Coupling with volume magnetic regions}

In this section, capacitive effects are neglected. We present how to adapt the proposed formulation in order to consider volume magnetic regions $\Omega_{\mathrm{M}}$ (see second problem presented in the section "Results"). In the context of a circuit representation, we have to consider a magnetic equivalent circuit for volume magnetic region which contains internal and external branches [2]. The magnetic fluxes flowing through internal magnetic branches are related to the flux density by the use of volume face elements, i.e.:

$$
\boldsymbol{B}=\sum_{j} \boldsymbol{w}_{v j} \Phi_{v j}
$$

where $\boldsymbol{w}_{s \boldsymbol{j}}$ is the volume face function associate to the face $\mathrm{j}$ of the volume discretization and $\Phi_{v j}$ the magnetic flux flowing through the $j$-th face. External branches connect nodes on the external surface of the volume magnetic region with the infinite, as in the case of regions treated by surface impedance condition. The previous equations can then be adapted by considering the fluxes $\left\{\phi_{s}\right\}$ and $\left\{\phi_{v}\right\}$ flowing in the magnetic regions, the external fluxes $\left\{\phi_{e}\right\}$ (treated by surface impedance or not) and surface currents $\left\{I_{s}\right\}$. We have:

$$
\{\phi\}=\left\{\begin{array}{c}
\phi_{v} \\
\phi_{s} \\
\phi_{e}
\end{array}\right\} \quad\{I\}=\left\{I_{s}\right\}
$$




$$
\begin{gathered}
{\left[Z_{I}\right]=[R+j \omega L] \quad\left[Z_{I \phi}\right]=\left[\begin{array}{lll}
j \omega C_{v} & j \omega C & 0
\end{array}\right]} \\
{\left[Z_{\phi I}\right]=\left[\begin{array}{c}
C_{v}^{\prime} \\
C^{\prime} \\
0
\end{array}\right] \quad\left[Z_{\phi}\right]=\left[\begin{array}{ccc}
Y_{v} & 0 & 0 \\
0 & Y & 0 \\
0 & 0 & Q
\end{array}\right]}
\end{gathered}
$$

with

$$
\begin{gathered}
Y_{v i j}=\iint_{\Omega_{M}} \frac{\boldsymbol{w}_{\boldsymbol{s i}} \boldsymbol{w}_{\boldsymbol{s}}}{\mu} d \Omega \\
C_{v i j}=\frac{1}{4 \pi} \int_{\Gamma} \boldsymbol{w}_{\boldsymbol{s i}} \iint_{\Omega_{M}} \frac{\mu_{r}-1}{\mu_{r}} \boldsymbol{w}_{\boldsymbol{v} \boldsymbol{j}} \wedge \boldsymbol{g r a d} \frac{\mathbf{1}}{\boldsymbol{r}} d \Omega d \Gamma \\
C_{v i j}^{\prime}=\frac{1}{4 \pi} \iint_{\Omega_{M}} \boldsymbol{w}_{v i} \int_{\Gamma} \boldsymbol{w}_{\boldsymbol{s j}} \wedge \boldsymbol{g r a d} \frac{\mathbf{1}}{\boldsymbol{r}} d \Gamma d \Omega
\end{gathered}
$$

Note that matrix Q must consider all the external faces of magnetic regions, treated by surface impedance condition or not. If two volume regions are in contact, a small change has to be done to determine $\mathrm{Q}$. Otherwise, we can notice that C and C' matrices need a volume integration on the magnetic region. However, previous works have shown that a relative coarse meshing of magnetic regions leads to good results when volume integral formulation based on circuit representation is used [12].

In order to get a good accuracy, the integration of Green's kernels of L, P, Q, C matrices are computed by using the analytical integration technique proposed in [3] for the self-interactions.

\subsection{Non simply connected domains}

It is worth notice that non-simply connected domains are naturally treated thanks to the use of a circuit solver (see the second example presented in the section "Results"). In order to efficiently obtain the incidence matrices $M_{I}$ and $M_{\phi}$, the circuit solver uses a specific algorithm, which combines the use of connectivity matrix between nodes and edges [6][7] of the primal mesh and independent loops search algorithm based on the circuit representation [5]. A first connectivity matrix is easily obtained from the finite element mesh and provides a set of small loops (i.e. the loops around each node) which is then provided to the circuit solver to be completed. Then, a specific algorithm builds independent loop matrices by adding missing loops and eliminating superfluous ones. This technic, based on [13], leads to an efficient determination of $M_{I}$ and $M_{\phi}$, with the advantages to prioritize small loops.

\subsection{Post-treatment}

The computation of the electric and magnetic fields inside the regions can be achieved tanks to the face finite element solution obtained for $\mathbf{K}$ and $\boldsymbol{\Phi}$. Fields in air and flux in coils can be obtained thanks to integral computed on the active regions [14]. Finally, thanks to the solution obtained for $\mathbf{K}$, losses are computed by integrating on $\Gamma$, i.e. 


$$
\text { Losses }=\frac{1}{2} \int_{\Omega} \frac{\boldsymbol{J} \overline{\boldsymbol{J}}}{\sigma} d \Omega=\frac{1}{2} \int_{\Gamma} \frac{\boldsymbol{K} \overline{\boldsymbol{K}}}{\sigma \delta} d \Gamma
$$

\section{Results}

We first present an academic problem composed of a conductive sphere (radius $\mathrm{R}=1 \mathrm{~m}, \mu \mathrm{r}=1, \sigma=5.5 \mathrm{E}+7 \mathrm{~S} / \mathrm{m}$ ) placed in uniform external field $\mathrm{B}=1 \mathrm{~T}$. Fig. 1 shows eddy current losses obtained by the analytical solution and the proposed PEEC SICBC formulation.

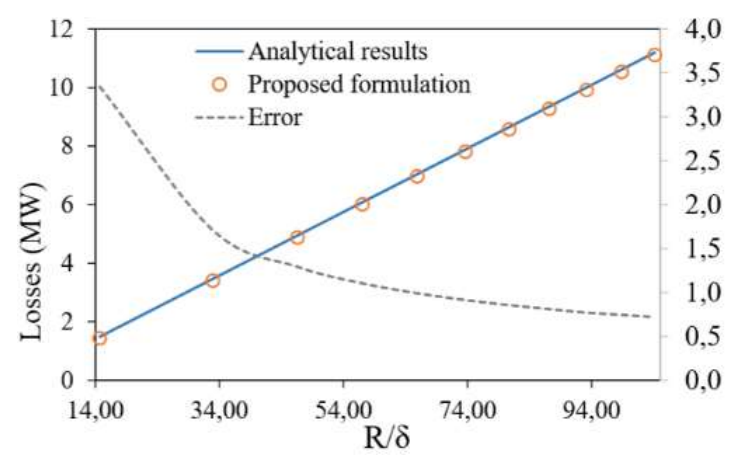

Fig. 4. Eddy current losses at different frequencies.

A more complex problem is considered with multiply connected regions as proposed by IEEJ (Fig.5) [4]. The conductivity and relative permeability of both plates are $5.5 \mathrm{E} 7 \mathrm{~S} / \mathrm{m}$ and $\mu_{\mathrm{r}}=100$, respectively. The ferrite region has a relative permeability of 3000 . The excitation coil is fed by an alternating current $1000 \mathrm{~A}, 1000 \mathrm{~Hz}$.
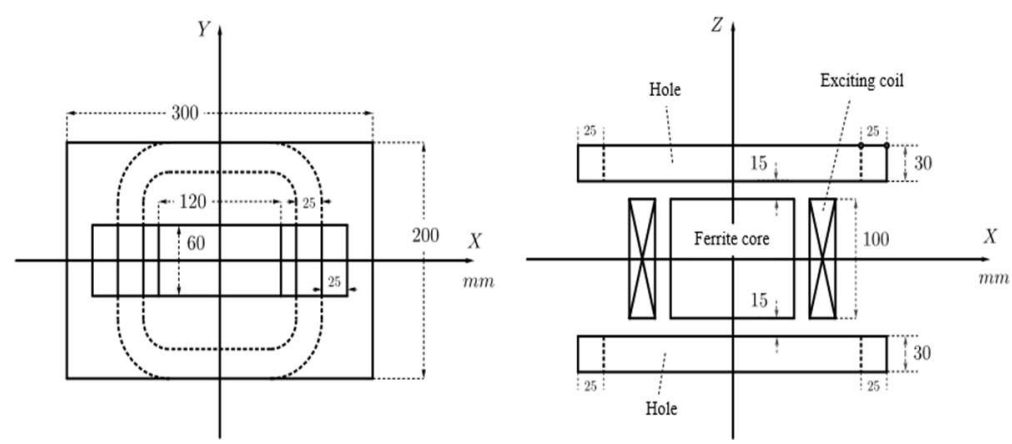

Fig. 5. The model proposed by IEEJ.
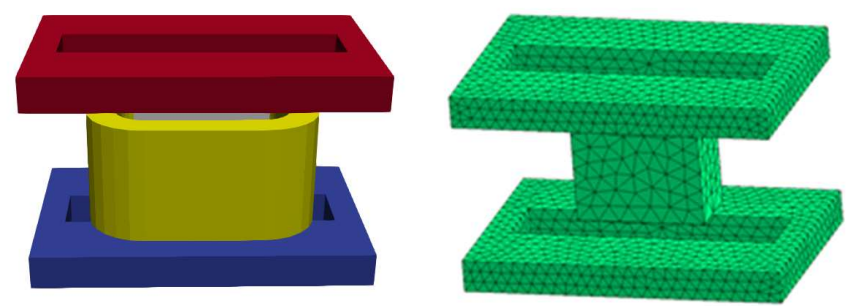

Fig. 6. Geometry and example of mesh for the model proposed by IEEJ 


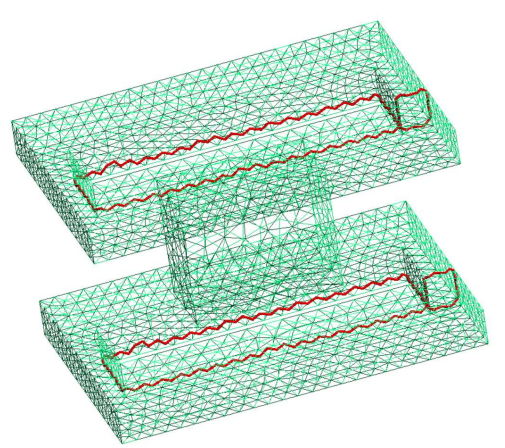

Fig. 7. Loops for taking into account non simply connected electric problem

In order to determine the independent loop matrices, the connectivity matrix from the primal mesh is used. For each conductive part, two supplementary loops are found by the circuit solver (Fig. 7) for the electric circuit representation, allowing to take into account the eddy currents flowing around the hole and along the section (Fig. 7), if any.

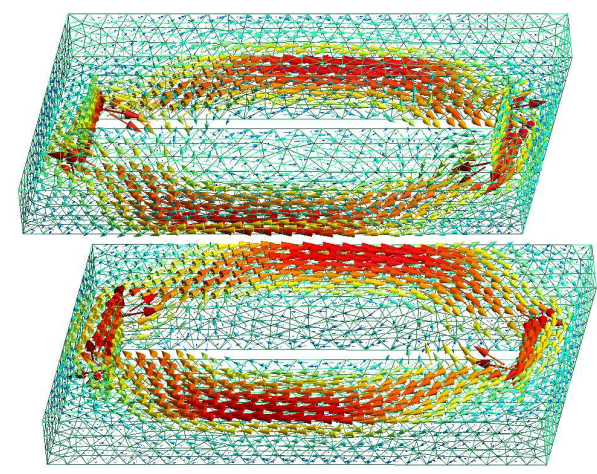

Fig. 8. Surface current $\mathrm{K}$ (real part) on conductive regions treated by SIBC

The density of the equivalent surface current K (Fig. 8) is compared with the result obtained by a FEM-SIBC formulation implemented in the Altair Flux ${ }^{\mathrm{TM}}$ software (Fig. 9). The difference in terms of Joule losses is about $0.04 \%$ for a fine mesh and of 1 $\%$ for a coarse mesh (Fig. 10).
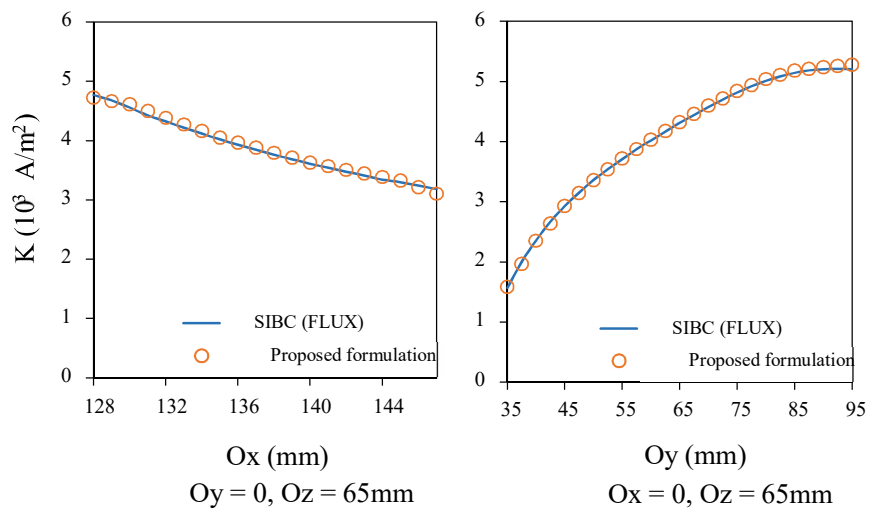

Fig.9. Comparison of the equivalent surface current K. 


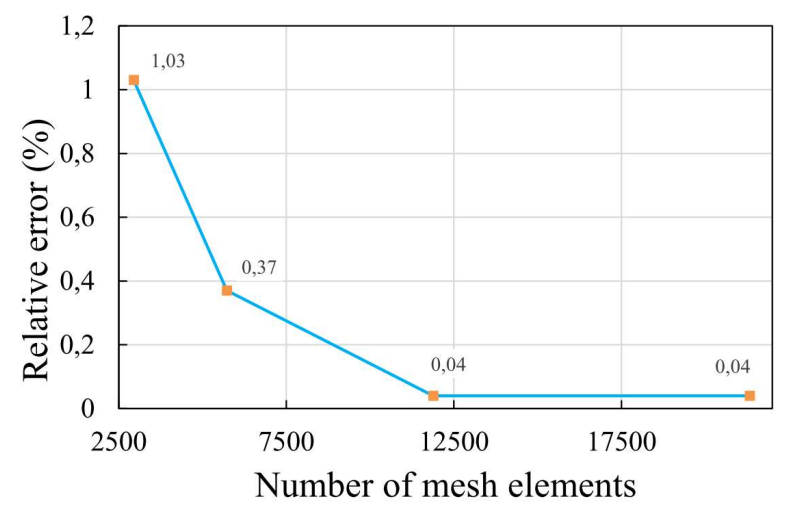

Fig.10 Comparison of losses between a FEM converged solution and the unstructured PEEC formulation. Both formulations use the SIBC condition.

\section{Conclusion}

The new surface impedance formulation enables the modeling of volume conductive and/or magnetic regions in order to efficiently simulate various devices with only a surface mesh. The computational effort is considerably reduced in comparison with volume approaches. The unstructured PEEC SIBC formulation has been tested in presence of volume magnetic nonconductive region and compared with a SIBC FEM approach.

\section{REFERENCES}

[1] Sergey Yuferev, Luca Di Rienzo, "Surface Impedance Boundary Conditions in Terms of Various Formalisms”, IEEE Trans-Mag, Vol 46, Issue 9, 2010.

[2] G. Meunier, O. Chadebec and J. M. Guichon, "A magnetic flux - electric current volume integral formulation based on facet elements for solving electromagnetic problems", IEEE Trans. Magn., vol. 51, no. 3, Mar. 2015.

[3] M. Fabbri, "Magnetic flux density and vector potential of uniform polyhedral sources", IEEE Trans. Magn., vol. 44, no. 1, pp. 3236, Jan. 2008.

[4] Nakata, T., Takahashi, N., Fujiwara, K., Imaj, T., Muramatsu, K. (1991) "Comparison of various methods of analysis and finite elements in 3-D magnetic field analysis”, IEEE Transactions on Magnetics, Volume: 27 , Issue: 5, Sept. 1991.

[5] T.-S. Nguyen, J.-M. Guichon, O. Chadebec, G. Meunier, and B. Vincent, "An independent loops search algorithm for solving inductive PEEClarge problems," Prog. Electromagn. Res. M, vol. 23, pp. 53-63, 2012.

[6] A. Bossavit and L. Kettunen, "Yee-like schemes on staggered cellulagrids: A synthesis between FIT and FEM approaches," IEEE Trans.Magn., vol. 36, no. 4, pp. 861-867, Jul. 2000.

[7] R. Specogna and F. Trevisan, "Eddy-currents computation with T- $\Omega$ discrete geometric formulation for an NDE problem," IEEE Trans.Magn., vol. 44, no. 6, pp. 698-701, Jun. 2008.

[8] R. Torchio, F. Moro, G. Meunier, J.-M. Guichon and O. Chadebec “An Extension of Unstructured-PEEC Method to Magnetic Media”, IEEE Trans-Mag, Volume: 55 , Issue: 6 , June 2019.

[9] Dalian Zheng, "Three-dimensional eddy current analysis by the boundary element method," in IEEE Transactions on Magnetics, vol. 33, no. 2, pp. 13541357, March 1997. doi: 10.1109/20.582507.

[10] R. Hiptmair, "Boundary element methods for eddy current computation, in Boundary Element Analysis" (Lecture Notes in Applied and Computational Mechanics), vol. 29. Berlin, Germany: Springer, 2007, pp. 213248.

[11] Z. De Grève, J. Siau, G. Meunier, J.M. Guichon, O. Chadebec, “A Mixed Surface Volume Integral Formulation for the Modeling of High Frequency Coreless Inductors”, IEEE Transaction on Magnetics, Year: 2016, Volume: 52, Issue: 3, Volume: 52, Issue 3

[12] Vinh Le-Van, Gérard Meunier, Olivier Chadebec, J.-M. Guichon "A Volume Integral Formulation Based on Facet Elements for Nonlinear Magnetostatic Problems", IEEE Transactions on Magnetics, Vol. 51, Issue 7, July 2015

[13] Joseph Douglas Horton, “A Polynomial-Time Algorithm to Find the Shortest Cycle Basis of a Graph”, SIAM Journal on Computing, April 1987. 
[14] Limin Huang, Gérard Meunier, Olivier Chadebec, Jean-Michel Guichon, Nicolas Galopin, Brahim Ramdane, “A Highly Efficient Post-Processing Method for Computing Magnetic Flux Considering Magnetic and Conductive Regions", IEEE Transaction on Magnetics, Volume 54, March 2018 